\title{
EL PESO DE LA PRESIÓN SOCIAL Y POLÍTICA: ESTUDIO DE LAS LIMITACIONES QUE CONDICIONAN EL DESEMPEÑO DE LOS PERIODISTAS EN CINCO PAÍSES LATINOAMERICANOS
}

The Weight of Social Pressure and Political: A Research on the Issues that Condition the Performance of Journalists in Five Latin American Countries

O peso da pressão social e política: estudo das limitações que condicionam o desempenho dos jornalistas em cinco países latino-americanos

GUTIÉRREZ ATALA, Fernando. Universidad Católica de la Santísima Concepción. (Chile) fgutierrez@ucsc.cl

ODRIOZOLA CHÉNÉ, Javier. Universidad de Los Hemisferios. (Ecuador) javiero@uhemisferios.edu.ec

FERREIRA, Javier. Universidad Autónoma de Bucaramanga. (Colombia) jferreira4@unab.edu.co

ANAYA ÁVILA, Pilar. Universidad Veracruzana. (México) pilargre@yahoo.com.mx 
PAJONI, Hernán. Pontificia Universidad Católica Argentina. (Argentina)

hernan_pajoni@uca.edu.ar

Fecha de recibido: 11 de agosto de 2015

Fecha de aceptado: 27 de octubre de 2015

\section{RESUMEN}

La comunicación presenta el primer informe de la investigación realizada por el grupo de estudios ERP (Estudio Rutinas Periodísticas), compuesto por académicos de Chile, Ecuador, Colombia, Argentina y México, orientado a identificar las limitaciones que afectan la labor informativa de los periodistas latinoamericanos. El documento permite conocer la relación entre los periodistas y el poder político, y las restricciones que este sector tiene en el acceso y difusión de la información. Asimismo, ahonda en la influencia de la audiencia en la reconstrucción de la realidad social, que también se configura como un efecto condicionante. De esta forma, y en la voz de sus propios protagonistas, se expone la compleja realidad laboral de los periodistas, observados tradicionalmente como agentes mediadores entre la agenda política y la agenda pública. La exposición de los resultados obtenidos permite determinar tendencias generales aplicables al conjunto de Latinoamérica y también realidades específicas de cada país y, a partir de allí, plantear cambios necesarios para asegurar que la profesión cumpla efectivamente su rol social.

Palabras clave: Periodismo, rutinas periodísticas, condicionantes, audiencias, presiones políticas, América Latina.

\section{ABSTRACT}

This text presents the first report of the research done by the ERP research team (Estudio Rutinas Periodísticas), integrated by academicians from Chile, Ecuador, Colombia, Argentina and México, oriented to identify the conditions that affect the informative labor of Latin American journalists. The document allows getting to know the relation between journalists and political power and the restrictions that this sector has in the access to and the broadcasting of information. Furthermore, the research deepens on the influence of audiences in the construction of social reality that also configures as a condition to information work. In this way, and in the voice of journalists themselves, often observed as intermediators between the politic and the public agenda, the complex labor reality of journalists is exposed. The exposition and comparison between the obtained results allows to determine general tendencies applicable to Latin America as a whole and also to specific realities in each country, and to propose the general changes needed to secure that the profession will be able to fulfill effectively its social role.

Keywords: Journalism, journalistic routines, conditions, audience, political pressures, Latin America. 


\section{RESUMO}

A comunicação apresenta o primeiro informe da pesquisa realizada pelo grupo de estudos erp Estudo de Rotinas Jornalísticas (Estudio Rutinas Periodísticas), composto por académicos do Chile, Equador, Colômbia, Argentina, e México, orientado a identificar as limitações que afetam o trabalho informativo dos jornalistas latino-americanos. O documento permite conhecer a relação entre os jornalistas e o poder político, e as restrições que este setor tem no acesso e difusão da informação. Igualmente, aprofunda na influência da audiência na reconstrução da realidade social, que também se configura como um efeito condicionante. Desta forma, e na voz de seus próprios protagonistas, se expõe a complexa realidade laboral dos jornalistas, observados tradicionalmente como agentes mediadores entre a agenda política e a agenda pública. A exposição dos resultados obtidos permite determinar tendências gerais aplicáveis ao conjunto de Latino-América e também realidades específicas de cada país e, a partir dali, apresentar mudanças necessárias para assegurar que a profissão cumpra efetivamente seu rol social.

Palavras-chave: Jornalismo, rotinas jornalísticas, condicionantes, audiências, pressões políticas, América Latina.

\section{Introducción}

En julio de 2014 se creó el grupo de investigación ERP (Estudio de Rutinas Periodísticas), compuesto por investigadores de Chile, Argentina, Ecuador, Colombia, Paraguay y México. Este grupo nació para estudiar las transformaciones que registran los mecanismos utilizados por periodistas latinoamericanos para recolectar, seleccionar y publicar información; las influencias que podrían afectar a dicho proceso, y los efectos que aquello tiene en el producto final que se ofrece al público.

EL ERP es un estudio de tipo cualitativo con un enfoque descriptivo/exploratorio. El primer informe, en plena etapa de análisis de la información recogida, estuvo orientado a la identificación de los condicionantes que los propios periodistas reconocen al autoevaluar sus rutinas de trabajo. Se elaboró sobre la base de 200 entrevistas semiestructuradas a informadores de Concepción (Chile), Buenos Aires (Argentina), Quito (Ecuador), Bucaramanga (Colombia) y Veracruz (México), representantes de cuatro soportes diferenciados: prensa escrita, radio, televisión y medios digitales.

La idea de fondo de este texto es determinar el peso específico de los condicionantes políticos y de presión social en el establecimiento de la agenda por parte de los periodistas latinoamericanos. Para lo anterior, se establecieron dos objetivos fundamentales: primero, determinar la percepción periodística sobre políticas comunicativas públicas en relación a la transparencia gubernamental y sus efectos y, en segundo lugar, identificar a la luz de lo expresado por los sujetos requeridos, prácticas periodísticas que revelen la importancia de la opinión ciudadana en la construcción de agenda. Con ello, se podrá determinar el peso de las influencias externas (políticas 
y de presión social) en la visión de los periodistas (ideología) en cada uno de los países y constatar si estos condicionantes se desarrollan de igual forma en todos ellos en una lógica supranacional o si responden a las propias realidades locales.

Es el interés del equipo ERP difundir los detalles de este estudio, reconociendo que el periodismo atraviesa una crisis multifactorial, ya casi permanente. La idea es aportar antecedentes sobre el porqué de esta etapa crítica que vive la profesión. Luego vendrán otros estudios y otros informes que serán un insumo concreto para contribuir desde la universidad a proveer de una radiografía profunda a la forma en que se está haciendo periodismo.

\section{Estado de la cuestión}

\subsection{El periodismo del siglo XXI}

Así como el convulsionado devenir de la sociedad actual, el periodismo como profesión no ha estado exento de los cambios surgidos. La vorágine con la que se desarrolla la actividad informativa en estos días es un reflejo del mundo del siglo xxı, donde la tecnología rompió gran parte de los paradigmas e impone nuevas formas de vivir, de comunicarse y de informar.

Ya en 1999, Martínez Albertos (1999) tenía una mirada retrospectiva y señalaba que antes en los periodistas destacaban valores como la información, el conocimiento y la determinación de las nociones de verdad y mentira. Sin embargo, "estos valores pueden quedar seriamente afectados en las próximas décadas como consecuencia derivada del impacto de las nuevas tecnologías aplicables a los fenómenos de la comunicación de masas” (p. 20). Este impacto queda en evidencia con la aceleración informativa actual, que convierte la inmediatez en una peligrosa obsesión, instalándola como objetivo prioritario y a la que deben supeditarse todos los demás valores de la información tradicionalmente respetados como el rigor, el contraste o la profundidad. Todos se subordinan a ella y "la feroz competencia entre los medios impide renunciar a la urgencia informativa", jibarizando el proceso comunicativo y convirtiéndolo en una competencia sin tregua (Serrano, 2014, p. 67).

Esta excesiva competencia está mutando de la era de los medios de masas a la era de "la masa de medios" (Ramonet, 2011, p. 22). Explica el autor, que de unos cuantos "medios-sol" en el centro del sistema, que determinaban la gravitación universal de la comunicación y de la información a su alrededor, ahora los "medios-polvo", diseminados por todo el sistema, son capaces de aglutinarse y amalgamarse para convertirse en súper plataformas mediáticas gigantes. Es decir, presenciamos el paso de un sistema media-céntrico a uno yo-céntrico, con el internauta como centro del mismo.

Desde toda perspectiva, la tecnología propició -y seguirá propiciando- un cambio en el sistema de comunicación, "desde el momento en que introdujo nuevas formas de creación colectiva de contenidos" (Alonso, 2010, p. 24). Todo decanta en las redes sociales, plataformas que permiten un interminable flujo de información de alta valoración social y comunitaria, pero que no necesariamente es originada por profesionales de la comunicación; esto actualiza y da sentido a la idea de una "mayor democratización de la información" (Ramonet, 2011, p. 23), pero al mismo tiempo da el punto de partida para una serie de riegos y desafíos de actualización y reperfilamiento de los profesionales de la información. 


\subsection{El periodismo en Latinoamérica}

Al hablar de Latinoamérica debemos tener en cuenta que "los países latinoamericanos son actualmente resultado de la sedimentación, yuxtaposición y entrecruzamiento de tradiciones indígenas (sobre todo en las áreas mesoamericana y andina), del hispanismo colonial católico y de las acciones políticas, educativas y comunicacionales modernas" (García-Canclini, 2001, p. 86). Con el asentamiento de la globalización cultural hay que tener en cuenta que las identidades ya no solo se construyen desde las raíces propias de cada cultura, haciendo que se deba tener en cuenta una nueva dimensión definida como moving roots, la cual hace referencia a las redes y flujos culturales fruto de migraciones y movilizaciones que desanclan la identidad cultural tradicional (Barbero, 2003, p. 21). De esta manera, se desarrollan comportamientos comunes entre sociedades con un desarrollo económico, cultural y político similar (Inglehart \& Carballo, 1997, p. 35). Sin embargo, si bien pueden presentar características comunes, también presentan características propias derivadas de la propia realidad nacional.

Esta cuestión también es aplicable al ejercicio del periodismo. El estudio de la figura del periodista a nivel latinoamericano involucra también el desafío de homogeneizar grupos culturalmente muy diversos. Sin embargo, esto posibilita la investigación comparativa al definirse "elementos comunes que han venido definiendo al periodismo en esta zona" (Mellado, 2009, p. 194). El punto de encuentro son, justamente, los condicionantes en la rutina laboral periodística abordados en esta investigación.

Un periodista no consiste en un autómata transmisor de contenidos, por el contrario, actúa en función de valores y paradigmas, y esta materia subjetiva deja adherencias en sus crónicas, aun cuando se esfuerce en ser imparcial y un invisible mensajero de la actualidad. "Por eso, en América Latina, el periodismo puede ser de alto o bajo nivel. Admirable, o execrable, pero solo en casos excepcionales logra ser objetivo” (Vargas Llosa, 1998).

Si bien cada país tiene sus particularidades en su relación medios-sociedad, el problema de los medios existe en todas las sociedades (McChesney, 2004, p. 16). Sin importar la época en la que se encuentre, el periodista latinoamericano desarrolla su trabajo acorde a su propia realidad. Después de más de treinta años del periodo de dictaduras latinoamericanas "existe la misma tensión entre aquellos que tienen el poder y los que no, la batalla solo asume diferentes formas. Los medios están en el centro de los conflictos por poder y control en cualquier sociedad"1 (McChesney, 2004, p. 17). Tal es el caso de países como Argentina o Ecuador donde el Estado vuelve a reposicionarse como el actor central de la regulación mediática y los medios (Guerrero \& Márquez, 2014, p. 144).

Pero, además, se registran cambios con motivaciones políticas o institucionales que han afectado las estructuras mediáticas y políticas de comunicación en cada país. Por ejemplo, en el caso de Chile y Colombia "las reformas mediáticas crearon condiciones para que el capital extranjero se aliara con corporaciones locales (...) o para hacerse adquirir por otros grupos mediáticos extranjeros" (Guerrero \& Márquez, 2014, p. 141). Trascendiendo las décadas, pareciera que en Latinoamérica es común que "aquellos en el poder generan un sistema de medios que apoya su dominación y minimiza [la] posibilidad de oposición efectiva”. Un ejemplo de ello es el caso de México, donde los propietarios y ejecutivos de los medios están jugando un papel cada vez más activo en la vida política (Márquez, 2014, p. 148). Todo esto se basa en la idea de que el uso de los medios de comunicación como principal

1 Traducción realizada por los autores del artículo. 
vehículo para hacer política y tomar al asalto los gobiernos "ya es una realidad en los países de economía. La casuística es variada, aunque similar en lo esencial" (Serrano, 2012, p. 22).

Este contexto socio-cultural que se puso en marcha en el siglo xx, sumado a la modernización de los procesos permite afirmar que en América Latina "la revolución tecnológica, la convergencia mediática y el papel del consumidor han transformado los ritmos y los modos en que se producen los mensajes, y con ello, las formas de expresión del periodismo" (Mellado, 2009, p. 197).

\subsection{Influencias en el ejercicio de la profesión periodística}

Los condicionantes en la práctica periodística han sido estudiados por diferentes autores, aportando diferentes categorizaciones que ayudan a determinar no solo los condicionantes en el desempeño de la profesión periodística, sino el grado de influencia de cada uno de ellos.

Para Shoemaker y Reese existen cinco niveles de influencia: a) la psicología del periodista; b) las rutinas periodísticas; c) las pautas organizacionales de los medios; d) las fuentes externas, y e) la ideología social predominante (Shoemaker \& Reese, 1996, pp. 63-105). Posteriormente, estas influencias han sido redefinidas como a) individuales, en relación al propio periodista como creador de los relatos periodísticos; b) rutinas periodísticas, asentadas en la profesión y, por tanto, tácitamente validadas; c) organizacionales, de acuerdo a los responsables de tomar decisiones en los propios medios de comunicación; d) instituciones sociales, donde se encuadran anunciantes, audiencias o grupos de interés, y e) el sistema, entendido como el sistema social o las ideologías de las sociedades (Shoemaker \& Vos, 2009, pp. 31-32).

Por su parte, el estudio dirigido por Hanizsch (2010) clasifica estas influencias en seis dimensiones: a) políticas, tanto de representantes políticos como de lobbys que se desenvuelven en el campo político; b) económicas, relacionadas con la necesidad de la publicidad como forma de financiar las empresas periodísticas; c) organizacionales, relacionadas con los procesos de toma de decisión en los medios de comunicación; d) de procedimiento, en relación a las limitaciones de tiempo y espacio con que trabajan los periodistas; e) profesionales, cuando se habla de las prácticas de trabajo asentadas de acuerdo a convenciones culturales de la profesión, y f) grupos de referencia, que determinan el trabajo del periodista y que están compuestos tanto por la audiencia como por otros actores diversos como compañeros de profesión, amigos o familiares (Hanitzsch et al., 2010, pp. 11-12).

Por último, cabe destacar, una última categorización de las influencias en la práctica periodística, que permite agruparlas en tres niveles: a) el de sistema, entendido como el marco social en el que se desenvuelven los medios de comunicación, relacionándose con otros sistemas de la sociedad; b) las influencias de la propia institución mediática, en su estructura y procedimientos habituales, y c) el periodista como actor que se relaciona con las rutinas de trabajo propias que el periodista desarrolla a nivel individual dentro del medio de comunicación (Oller \& Meier, 2012). De acuerdo con los expuesto hasta el momento, la influencia contextual del trabajo periodístico puede estudiarse desde los sistemas, la institución y el actor (Oller \& Chavero, 2015, p. 89).

Para concluir, hay que tener en cuenta que las influencias no se desarrollan horizontalmente sino que se establecen siguiendo una jerarquía "que propone importantes distinciones entre los niveles de análisis y sitúa al periodista individual en una red de limitaciones organizacionales e ideológicas” (Reese, 1999, p. 48). Así, los condicionantes ideológicos de los periodistas se ven limitados por condicionantes institucionales, relacionados con 
la organización jerárquica de los medios y las rutinas periodísticas, que a su vez dependen del contexto social en el que los profesionales de la información y los medios se desenvuelven.

\subsubsection{Las influencias políticas}

La configuración de la agenda mediática viene marcada, tradicionalmente, por la prevalencia de los acontecimientos políticos y de sus protagonistas como fuentes de información (Gandy, 1982; Manheim, 1994; Kennamer, 1994). Esta centralidad de la actividad política, según palabras de Grossi (1985), "se encuentra intrínsecamente conectada a la facultad de otorgar relevancia pública a un evento, de conferirle importancia en el plano social, de ubicarlo, en tanto que es una cuestión significativa, en nuestra vida cotidiana" (Grossi 1985, citado por Casero, 2006, p. 181).

Este hecho se ve fortalecido por la propia práctica periodística (Rodrigo-Alsina, 2005; Domingo, 2006) de tal manera que "la prominencia de las fuentes del Gobierno determina todo el proceso de acceso, selección y producción informativa" (Pérez, 2008, p. 253), pues, al identificar a las fuentes centralizadas de información como instituciones sociales legitimadas, "estos sitios pasan a ser objetivados como los sitios apropiados donde ha de recogerse la información. Así, mediante la recolección de noticias son objetivados como las fuentes legítimas y legitimadoras de la información" (Tuchman, 1983, p. 224).

Ante esta situación, la relación entre periodistas y agentes políticos se configura en torno a dos ejes, conflicto/ cooperación e independencia/dependencia, produciendo cinco posibles modelos de relación entre periodistas y políticos: a) adversarial, entendiendo los medios como un cuarto poder que ejerce una intensa labor de vigilancia; b) colateral, que se caracteriza por presentar puntos de vista similares a los de las instituciones políticas; c) de competición, en el que medios de comunicación y políticos pugnan por controlar los problemas centrales de la agenda pública; d) de intercambio, que afirma que debido a la mutua necesidad, ambos actores prefieren el acuerdo y la colaboración tácita, y e) de negociación constante, caracterizada por una interacción constante, altamente dinámica y en perpetua mutación (Casero, 2008, pp. 105-113).

\subsubsection{Las influencias de la audiencia}

Históricamente, la relación entre periodistas y audiencia se ha configurado desde una visión unidireccional. Autores como Laswell (1948) plantean un modelo de comunicación centrado en cinco elementos básicos: quién dice, qué dice, en qué canal, a quién y con qué efecto. Posteriormente, este planteamiento ha sido criticado puesto que "deja el control del acto comunicativo en manos del emisor mientras que la audiencia es manipulable y pasiva" (Iguartua \& Humanes, 2004, p. 114). Así, desde la semiótica, se afirma que, pese a que el mensaje es único, las posibles decodificaciones del mismo que hacen los receptores son múltiples (Eco \& Fabbri, 1978, p. 571). Frente a esta propuesta nos encontramos con el modelo bidireccional, en el que se da una retroalimentación entendida como "la información que proviene, en retroceso, del receptor al transmisor, y le indica a este cómo se desarrolla su mensaje" (Scharmm, 1982, p. 16).

Tradicionalmente, la retroalimentación en la comunicación masiva ha sido escasa (Scharmm, 1982, p. 16). Sin embargo, con la aparición de las nuevas tecnologías, los medios de comunicación han podido evolucionar hacia un modelo con una mayor participación de las audiencias. Así, gracias a la interactividad comunicativa se amplían 
las posibilidades de comunicación y expresión de las audiencias hacia los propios medios de comunicación (Rost, 2014, p. 55). La ocupación por parte de medios de comunicación y periodistas de las redes sociales, ha permitido mejorar la comunicación directa entre estos y sus audiencias. Sin embargo, diferentes investigaciones vienen demostrando que los medios todavía tienen dificultades para conectar con la audiencia y generar comunidad (Messner, Linke \& Eford, 2011; García De Torres et al., 2011). Por tanto, los periodistas siguen teniendo su propia concepción sobre los intereses de sus audiencias, pese a que, hoy en día, es posible una comunicación directa con las mismas.

\section{Metodología}

La herramienta metodológica utilizada para esta ponencia fue la entrevista semiestructurada y se centró en extraer resultados desde dos perspectivas. En primer lugar, por medio de una entrevista de respuesta espontánea, se indagó en la determinación de la presencia de condicionantes políticas y de presión social que marcan la labor periodística. En segundo lugar, se realizó un análisis mediante respuesta sugerida, que permitió profundizar en los aspectos concretos planteados por los entrevistados.

Para ello, se realizaron 200 entrevistas a periodistas en activo en cada uno de los subgrupos nacionales (40 por país y 10 en cada soporte: prensa escrita, radio, televisión, medios digitales). Luego, las respuestas fueron transcritas y ordenadas en tablas sometidas a análisis por los investigadores, previa aplicación de la prueba de fiabilidad intercodificadores.

La fiabilidad es una condición necesaria para la validación de cualquier estudio. Aunque no la garantiza, sí establece límites a la validez potencial de la información recabada. Actúa como salvaguardia contra la contaminación de los datos por efectos ajenos a las finalidades de observación, medición y análisis (Krippendorff, 1990, pp. 191-192). Es decir, otorga la seguridad de que los datos han sido obtenidos con independencia del suceso, del instrumento o la persona que los mide.

Los datos sobre fiabilidad exigen que dos codificadores, como mínimo, describan en forma independiente los registros en un lenguaje común, para comprobar si efectivamente se da la intersubjetividad y el grado de acuerdo entre los codificadores participantes. Para este estudio, se consideraron dos codificadores. Una vez ejecutado el trabajo de ambos, la fiabilidad de los resultados arrojados por sus análisis se calcula a través del procedimiento para datos nominales denominado Método de Holsti, cuya fórmula de fiabilidad es una de las más utilizadas en estudios de características similares (Igartua, 2006, p. 221). El estudio utiliza este método, que no considera el acuerdo por azar, puesto que no solo se mide la presencia de los condicionantes, sino que codifica cada uno de los segmentos de la respuesta espontánea. Así, un condicionante puede ser nombrado varias veces por cada entrevistado.

Consideraremos que Fiabilidad $=2^{*} M \div(N 1+N 2)$, donde $M$ representa el número de decisiones de codificación en la que dos codificadores coinciden, mientras la suma de N1 y N2 expresa el total de decisiones de codificación de ambos codificadores. Un acuerdo en todas las decisiones daría el resultado 1. En este estudio, un 10\% del universo fue revisado (20 entrevistas) y seleccionado de manera aleatoria, asegurando la representatividad de los 5 países involucrados en el estudio. 
Así, para las condicionantes políticas tenemos: $2^{\star} 18 \div(20+20)$, lo que nos otorga un grado de fiabilidad de 0,9 . Para los condicionantes de presión social los datos son: $21^{\star} 17 \div(20+20)$, datos con los que la prueba de fiabilidad arroja un 0,85 . Si se establece que el nivel de acuerdo mínimo que se debe alcanzar para considerar que los datos son fiables es de 0,8 (80\%) (Igartua, 2006, p. 221), podemos considerar que nuestro estudio es altamente fiable con un resultado promediado de acuerdo entre codificadores de un $87,5 \%$.

\section{Resultados}

\subsection{Resultado de las entrevistas a periodistas de Concepción (Chile)}

A continuación, se exponen y explican las respuestas y opiniones mencionadas por los sujetos numerados desde el 1 al 40 correspondientes a Chile.

Tabla 1. Presencia de los condicionantes en las entrevistas a periodistas chilenos

\begin{tabular}{|l|c|c|}
\hline \multicolumn{1}{|c|}{ Chile } & $\begin{array}{c}\text { Entrevistas } \\
\text { realizadas }\end{array}$ & $\begin{array}{c}\text { Casos } \\
\text { reconocidos }\end{array}$ \\
\hline Condicionantes Políticas & 40 & 6 \\
\hline Condicionantes de Presión Social & 40 & 15 \\
\hline
\end{tabular}

Fuente: Estudio sobre Rutinas Periodísticas.

En lo referente a las presiones políticas, en primer lugar, es común que existan casos en los que se les ordena a el o la periodista cubrir información de partidos políticos sin ningún motivo coherente o fundamentado en la contingencia. En segundo lugar, los personeros de la esfera política telefonean al profesional de la comunicación que escribió algo que no les favorece para quejarse al respecto. "En algunos casos se llega incluso a descalificar al autor de la noticia" (Sujetos 7 y 21). También hay una insistencia a los medios de comunicación para que cubran sus actividades. La insistencia, según se afirma, "es extrema en algunos casos" (Sujeto 27). Estas presiones llegan incluso a ser personalizadas para que no se publique información ya reporteada (Sujeto 1). En tercer lugar, es una práctica común de personeros políticos el entrar en dinámicas de negociación con los periodistas. Ofrecen "soltar" información exclusiva a cambio de favores (Sujeto 4). En cuarto lugar, es posible también que políticos enemigos consideren a los medios como abanderados de su competencia si se publica sobre esta de manera, relativamente, constante. El político puede llegar a advertir que "dejará de proveer información al medio" (Sujeto 20). En quinto lugar, se menciona como un requisito para lograr un periodismo de calidad "la necesidad de un marco legislativo que permita asegurar condiciones básicas para hacer un buen trabajo y evitar un desamparo laboral” (Sujeto 33). En sexto lugar, el Código del Trabajo establece una cantidad de horas de trabajo máximas, pero esto no se aplica en algunos contratos que deben firmar los periodistas para ingresar a trabajar en medios y que, por lo tanto, "los somete a horarios de trabajo ambiguos y sin límites definidos” (Sujeto 17). En séptimo y último lugar, ya en el plano del ejercicio, los equipos que asesoran comunicacionalmente a las figuras públicas se presentan, en algunos 
casos, como obstáculos para el desarrollo de la labor periodística (Sujeto 12). Y en casos puntuales, las fuentes oficiales (Gobierno) discriminan a los periodistas de medios que consideran "poco serios" y, por lo tanto, les dificultan el acceso a la información (Sujeto 5).

En cuanto a los condicionantes relacionados con la presión social y las audiencias; en primer lugar, se evidencia que agrupaciones sociales de distinta índole creen que los medios están a su servicio y suelen dar instrucciones al periodista para que cubra sus informaciones. En caso de negarse se suele culpar al periodista de trabajar para la "burguesía" (Sujeto 21). En segundo lugar, cabe destacar que, según la plataforma informativa, la influencia de las audiencias puede variar. Por ejemplo, en el contexto radial la demanda ciudadana siempre ha sido fuerte. Este fenómeno se vio incrementado drásticamente luego del terremoto del 27 de febrero de 2010, "cuando la radio cumplió un rol social importante" (Sujeto 28). Ahora los periodistas se enfrentan a una marea de personas que "creen tener contenido noticioso cuando no es el caso". Esto ocurre en cualquier sitio, tanto en oficinas como en la calle (Sujetos 27 y 29). En tercer lugar, los temas sociales pueden "aparecer espontáneamente y modificar la agenda" en algunos medios (Sujeto 5), lo que ha llevado a pensar a la gente que sus problemáticas deben ser publicadas de forma instantánea por el hecho de proveer información, aunque esta no tenga factores noticiosos. Esto se da especialmente en localidades más pequeñas y en medios digitales (Sujetos 10 y 11). En cuarto lugar, en medios digitales, las críticas (comentarios) son crudas y despiadadas. "Sin importar la calidad de la elaboración, si el tema no es de agrado del público, se realizan críticas a destajo e incluso, a veces, sin fundamento". Se destaca el carácter violento de las opiniones (Sujetos 11 y 14).

\subsection{Resultado de las entrevistas a periodistas de Bucaramanga (Santander, Colombia)}

En el siguiente apartado se exponen y explican las respuestas y opiniones mencionadas por los sujetos numerados desde el 41 al 80, correspondientes a Colombia.

Tabla 2. Presencia de los condicionantes en las entrevistas a periodistas colombianos

\begin{tabular}{|l|c|c|}
\hline \multicolumn{1}{|c|}{ Colombia } & Entrevistas realizadas & Casos reconocidos \\
\hline Condicionantes Políticas & 40 & 14 \\
\hline Condicionantes de Presión Social & 40 & 15 \\
\hline
\end{tabular}

Fuente: Estudio sobre Rutinas Periodísticas.

En lo referente a las presiones políticas, el periodismo independiente se ve condicionado por el pensamiento editorial (Sujeto 74). Según los propios periodistas "se debe ser menos agresivo con lo que se piensa respecto a la gobernación o alcaldía” (Sujeto 79) porque se sabe que "los políticos amenazan a los periodistas cuando se ven involucrados en investigaciones realizadas por el medio de comunicación" (Sujeto 47). Sumado a esto, al ser el medio una empresa privada, que tiene una ideología política marcada, "no se pueden tocar ciertos temas" (Sujeto 52). 
En segundo lugar, "el gobierno pretende que los medios repliquen sus mensajes" (Sujeto 77), pero ocurre también que por publicar ciertas informaciones de algún partido político, son tachados como oficialistas y los del partido contrario no hablan con ese medio" (Sujeto 43). En tercer lugar, la política afecta los presupuestos de los medios, los políticos "contratan amigos o personas afines a sus campañas y se les suministra [información] en función de eso" (Sujeto 77). "No puedes hablar mal de un gobernador o alcalde o quedas marcado negativamente, y a raíz de eso no dan más publicidad" (Sujeto 66). Los políticos han creado un sistema en el que el periodista paga por trabajar y en consecuencia "se ha ido perdiendo la calidad informativa" (Sujeto 64) por lo que "se ha perdido el enfoque periodístico a raíz de la influencia” (Sujeto 61).

En cuanto a los condicionantes relacionados con la presión social y las audiencias, en primer lugar, los periodistas señalan que existe violencia e intolerancia por parte de las personas (Sujeto 43) que creen "que los periodistas son sobornados para no hablar mal de los políticos" (Sujeto 47). Además, existen "presiones marcadas por las clases sociales" (Sujeto 45). En segundo lugar, las audiencias son muy exigentes: "si no hay buen periodismo no hay audiencia" (Sujeto 65) pero al mismo tiempo, se siente atraída por el morbo y si este no está presente "el rating baja" (Sujetos 75 y 80 ).

\subsection{Resultado de las entrevistas a periodistas de Buenos Aires (Argentina)}

A continuación, se exponen y explican las respuestas y opiniones mencionadas por los sujetos numerados desde el 81 al 120, correspondientes a Argentina.

Tabla 3. Presencia de los condicionantes en las entrevistas a periodistas argentinos

\begin{tabular}{|l|c|c|}
\hline \multicolumn{1}{|c|}{ Argentina } & Entrevistas realizadas & Casos reconocidos \\
\hline Condicionantes Políticas & 40 & 11 \\
\hline Condicionantes de Presión Social & 40 & 8 \\
\hline
\end{tabular}

Fuente: Estudio sobre Rutinas Periodísticas.

En lo referente a las presiones políticas, los periodistas saben de manera interna cuáles son los temas de los que no se puede hablar (Sujetos 103 y 105) y reconocen enfrentamientos ideológicos con las autoridades (Sujeto 84). Además, se ha naturalizado que "hacer periodismo es hacer oposición del gobierno, en cualquier cobertura o tema" (Sujeto 89). En segundo lugar, "o estás de algún lado político o ideológico o te involucran con alguno" (Sujeto 109), "las líneas editoriales de los medios están definidas por quienes están a favor o en contra del gobierno, pero existen temas que no pueden censurarse" (Sujeto 101). En tercer lugar, "hay amistades por conveniencia, hay periodistas rentados por dirigentes o empresarios, hay información a cambio de silencios" (Sujeto 83). En consecuencia, los periodistas buscan equilibrar los intereses políticos dentro de alguna nota para evitar molestias (Sujeto 87) porque "reconocen que sus libertades dependen de la interacción con las partes políticas comprometidas en las notas" (Sujeto 88). En cuarto lugar, es una opinión transversal en diferentes medios que el gobierno significa, en 
Argentina, una presión constante. En el caso específico de la televisión, se reconoce que en los últimos años no trabajaron cómodos por presiones editoriales y gubernamentales (Sujeto 113). Del mismo modo, una radio específica se definió contraria al gobierno, "por lo que las noticias negativas serán siempre prioritarias" (Sujeto 110). En quinto lugar, los periodistas confiesan que el llamado telefónico del político es el modo que más evidencia algún condicionamiento (Sujeto 112), pues los políticos llaman siempre a los periodistas, "hasta por situaciones insustanciales" (Sujeto 82).

Con respecto a los condicionantes que provienen de la presión social, se reconoce una notoria presencia a partir de la activación de las audiencias pues "el periodismo ciudadano pasó a tener un lugar muy importante. Son un nuevo productor que te avisa dónde está la noticia" (Sujeto 115) y "las redes sociales ayudan mucho a medir el impacto de un tema y a actuar en consecuencia" (Sujeto 117). De todas formas, vinculado al nuevo entorno tecnológico o no, la figura del auditor/consumidor de información constituye una figura omnipresente que determina las decisiones editoriales e informativas que el medio toma:

"Cada redacción que escribo es para comunicar a un lector que me imagino que necesita tales o cuales aclaraciones" (Sujeto 82), "el criterio principal parta la definición de contenidos es por el espectador-tipo que define el noticiero. Cada editor de contenido decide lo que va y lo que no en función de eso" (Sujeto 111), y “QQué quieren nuestros oyentes? Es una pregunta que me hago siempre" (Sujeto 107). Aunque no todos lo reconozcan como una condición, se considera al espectador-tipo "una potencia para dirigir nuestro trabajo hacia ese diálogo imaginado con él” (Sujeto 84). En un nivel menor, aparecen presiones sociales vinculadas a la política: "tenemos la presión de que nos leen quienes [se] adhieren a nuestra postura" (Sujeto 81), y a la meta autoimpuesta por la pluralidad "nos imponemos cumplir con el respeto a la diversidad y el respeto de las identidades regionales" (Sujeto 119).

\subsection{Resultado de las entrevistas a periodistas de Quito (Ecuador)}

Este apartado expone y explica las respuestas y opiniones mencionadas por los sujetos numerados desde el 121 al 160, correspondientes a Ecuador.

Tabla 4. Presencia de los condicionantes en las entrevistas a periodistas ecuatorianos

\begin{tabular}{|l|c|c|}
\hline \multicolumn{1}{|c|}{ Ecuador } & Entrevistas realizadas & Casos reconocidos \\
\hline Condicionantes Políticas & 40 & 22 \\
\hline Condicionantes de Presión Social & 40 & 8 \\
\hline
\end{tabular}

Fuente: Estudio sobre Rutinas Periodísticas.

En lo referente a las presiones políticas, tres aspectos destacan en las respuestas: las presiones políticas que aparecen en el ejercicio diario de la profesión, el nuevo entorno jurídico que surge con la aprobación de la Ley Orgánica de Comunicación y los problemas de acceso a las fuentes gubernamentales. A la hora de fijar las presiones políticas, algunos entrevistados reflejan el "temor a ser perseguido, enjuiciado y sancionado" (Sujeto 139), creando una 
institucionalidad restrictiva, sancionadora y enajenante que busca poner a los funcionarios por encima del trabajo de los periodistas (Sujeto 138). Estas presiones se traducen en "campañas enteras para desprestigiar a periodistas que hacen bien su trabajo (...) existe un monitoreo continuo de todos los medios de comunicación o por lo menos de un altísimo porcentaje" (Sujeto 141) y "sistemáticas estrategias políticas de comunicación y marketing, con el objeto de afectar la noble misión de informar y entretener" (Sujeto 150). La consecuencia última sería que "las presiones políticas que han hecho que varios medios de comunicación cierren, vendan o desaparezcan” (Sujeto 148). Pese a ello, los periodistas no creen que este tipo de presiones logren determinar la práctica periodística: "a veces recibimos cartas cuestionando tal o cual noticia, pero no tienen mayor efecto en la cobertura, porque ahí se ve el prestigio de los medios" (Sujeto 146). Cabe destacar, que la práctica total de estas afirmaciones, en relación a las presiones políticas se efectúa desde periodistas que trabajan en medios privados.

Otro de los aspectos más mencionados por los periodistas dentro de las presiones políticas es la Ley Orgánica de Comunicación, vigente en Ecuador desde el 24 de junio de 2013. Se constata que puede darse un efecto negativo "por el desconocimiento de las leyes que atemoriza a los periodistas" (Sujeto 136) y se afirma que "la ley termina siendo una ley subjetiva en muchos aspectos o de enfoque subjetivo, que determina sanciones y limitaciones para el ejercicio periodístico" (Sujeto 154). Sin embargo, los periodistas entrevistados también consideran que esta ley tiene efectos positivos, pues potencia "ser mucho más responsables en la información que se entrega" (Sujeto 143) y realizar un mayor esfuerzo periodístico pues "obliga el tema de tener todas las fuentes posibles" (Sujeto 151). En definitiva "lo que te exige es ser más responsable con la forma en la que informas" (Sujeto 152). Por último, los periodistas ecuatorianos destacan las dificultades de acceso a las fuentes institucionales. Estos problemas se derivan de una problemática mundial puesto que "existen fuerzas políticas, existe una manipulación de la información en general, en el mundo entero" (Sujeto 158). En Ecuador, "es muy complicado tener la fuente oficial" (Sujeto 159). Así, "se trata más de un tema de censura previa, o sea, estamos claros en que el Gobierno no habla, no da entrevistas, entonces se vuelve complicado hacer temas que necesariamente requieren contrastación" (Sujeto 129). Pese a ello, también se afirma que si el acceso a las fuentes oficiales fuera más difícil, ya hubiesen muerto todos los medios, "ahora puedes mandar una carta o hacer una llamada. Es mucho más fácil. Puedes hablar con especialistas, poner una nota (...)" (Sujeto 131).

En cuanto a los condicionantes relacionados con la presión social y las audiencias; en primer lugar, desde la profesión periodística ecuatoriana, se perciben intentos de presión desde "todo lado" (Sujeto 128), tanto "las organizaciones públicas como las privadas tratan de darte el enfoque” (Sujeto 125). Sin embargo, los periodistas consideran que pese a que su trabajo sufra presiones de diferentes ámbitos "la presión más fuerte es la de la opinión pública. La gente nos exige que se publique la información” (Sujeto 137). En este punto también se reflejan las diferentes perspectivas de cómo deben actuar los periodistas para cumplir esta función social. La primera perspectiva construye sus relatos periodísticos teniendo en cuenta que "se trabaja para el público y sus intereses, nuestra obligación es interpretar esos intereses, saber qué es lo que la gente quiere saber para satisfacer esa necesidad" (Sujeto 154). Desde esta perspectiva, el no tener en cuenta los intereses de la audiencia puede provocar "un proceso paulatino de desclasamiento que los aleja de la sociedad en su conjunto y tratan de ubicarse en una escala en la que están las autoridades públicas, los actores sociales reconocidos..." (Sujeto 153). Desde la perspectiva contraria se afirma que "hay temas que la gente debe saber aunque no los quiera" (Sujeto 134). De esta manera, el periodista debe ejercer de filtro intelectual puesto que "no te puedes dejar llevar por la presión 
porque a veces la presión social puede dibujarse como un error y tú cometes el error (...) A veces tienes presión de por qué no dices esto, por qué no informas esto, deberías denunciar esto... pero no tienes las bases, no tienes los argumentos" (Sujeto 155).

\subsection{Resultado de las entrevistas a periodistas de Veracruz (México)}

Esta sección expone y explica las respuestas y opiniones mencionadas por los sujetos numerados desde el 161 al 200, correspondientes a México.

Tabla 5. Presencia de los condicionantes en las entrevistas a periodistas mexicanos

\begin{tabular}{|l|c|c|}
\hline \multicolumn{1}{|c}{ México } & Entrevistas realizadas & Casos reconocidos \\
\hline Condicionantes Políticas & 40 & 29 \\
\hline Condicionantes de Presión Social & 40 & 10 \\
\hline
\end{tabular}

Fuente: Estudio sobre Rutinas Periodísticas.

Con relación a las presiones políticas, hay una visión de los medios como empresa, y se presenta como obvia la subordinación a intereses políticos (Sujeto 163). En primer lugar, un gran número de periodistas afirma una influencia ideológica, religiosa y política en cuanto a los contenidos que se publican (Sujetos 162 y 164). Esto se debe principalmente "a convenios con el Gobierno que generan intereses a los que se debe responder". Además, se menciona la gran variedad de medios de comunicación como una estrategia del Gobierno para que hablen bien de él (Sujeto 177). Hay dependencia editorial y un "clientelismo político" (Sujetos 168 y 173); se trata de una relación paradójica ya que, según los mismos periodistas, "te capacitan y luego te critican" (Sujeto 175). En segundo lugar, "Las fuerzas que influyen en la toma de decisión sobre la información que se publica son principalmente las políticas y las económicas" y se menciona un caso específico: "Llevo dos agresiones físicas, un despido y varias amenazas, siempre por el estilo de indagar, pues me gusta exhibir actos de corrupción o malas acciones, y no es la delincuencia organizada la responsable, es el Gobierno el principal hostigador de los periodistas" (Sujeto 182). Otros periodistas agregan que "en [los] medios se corren todo tipo de riesgos, ya sea desde ser corrido por la empresa hasta la agresión por parte del gobierno" (Sujeto 193) y a simple vista, la corrupción y la empatía por ideologías políticas condicionan los contenidos de la agenda "por encima de la línea editorial del medio" (Sujeto 188).

En cuanto a los condicionantes relacionados con la presión social y las audiencias, los profesionales de la comunicación mencionan un totalitarismo de las audiencias que condiciona su rutina (Sujeto 172), porque la gente busca buena información (Sujeto 183). Hay una crítica directa de parte de la sociedad hacia los periodistas, se afirma que son "corruptos y vendidos". Por esta imagen es que los periodistas mexicanos son catalogados como "chismosos o estafadores" (Sujeto 178). 


\section{Discusión y conclusiones}

En términos generales, los periodistas consultados responden a una realidad común negativa y estructural a la hora de hablar de transparencia para el periodismo, pues en todos los casos aparece - con distintos grados de intensidad - la carencia de un acceso expedito, seguro y real a fuentes de gobierno sin las cuales es imposible cumplir adecuadamente y de manera prolija la función informativa (y por ende social) que se espera de los medios de comunicación.

Pese a lo anterior, aparecen matices particulares en la situación de cada país, fruto de la interpretación del relato de los profesionales consultados, que puede resumirse en las siguientes ideas. En Chile, las principales dificultades nacen de la estructura burocrática que tiene como consecuencia una disfunción del sistema, presionado por negociaciones y amiguismo político a la hora de conseguir información de fuentes oficiales. Al mismo tiempo, se detectó el problema opuesto, cuando personeros políticos necesitan visibilidad, en cuyo caso, la restricción de información es reemplazada por saturación de la misma, pero solo en temas de interés para el demandante. Esta situación se da en todos los soportes informativos.

El terreno de las audiencias pasa por un periodo de notoria actividad en un contexto de web 2.0. La democratización de la tecnología en Chile y el fácil acceso al debate público a través de casillas de comentarios, deja al periodista expuesto a opiniones, en muchos casos, infundadas, crudas e incluso anónimas, lo que es ampliamente reconocido por los periodistas como un importante factor de desmotivación laboral.

En Argentina, aparece un sistema burocrático similar al chileno, pero altamente potenciado por la polarización y la efervescencia política, que genera un sistema informativo muy ideologizado con una dinámica de bandos y rivalidades omnipresente en todo el proceso de generar y transmitir noticias, en las cuales siempre subyace un matiz político-partidista. A ese contexto se suma la alta tensión en las salas de redacción, donde la presión editorial es reconocida como fuerte. Los resultados arrojados por los sujetos argentinos no explicitan una realidad tan extrema como en otros países latinoamericanos. Sin embargo, a pesar de carecer de un ambiente de extrema violencia o miedo, existen factores condicionantes, que se encuentran en el terreno de las prácticas integradas y aceptadas como normales.

Las audiencias se presentan también como un factor de presión, pero desde una perspectiva interna en la que el periodista argentino escribe para un lector ideal. Aún en un contexto altamente politizado, no hay evidencias de que las audiencias se manifiesten de forma condicionante.

En Colombia, el principal problema es la deslegitimación legal de la función periodística, que se traduce en la proliferación de "periodistas empíricos" y la restricción al acceso a la información oficial. En forma paralela, los periodistas coartan su accionar producto de la influencia del poder político que se traduce ocasionalmente en amenazas directas o indirectas, debido a que muchos de los medios tienen dependencia gubernamental. Otros medios, aunque privados, también responden a una dinámica de negociación política.

A pesar de que Colombia también presenta un alto nivel de efervescencia política, además de un conflicto armado que lleva más de 50 años, los periodistas no reconocen los conflictos políticos como el mayor condicionante. Si bien existe un miedo latente al investigar temas con conflicto de intereses, los periodistas colombianos mantienen su preocupación en detalles que les impiden realizar eficazmente sus labores cotidianas de reporteo. 
La presión de las audiencias incide en los niveles de rigurosidad. Los mismos sujetos afirman que el público exige contenido de calidad. Pero, aunque selectiva, la audiencia no se consolida como una fuerza esencialmente condicionante, a pesar de existir los medios tecnológicos para que esto ocurra.

En Ecuador, al igual que ocurre en Colombia, los periodistas reflejan una falta de transparencia informativa de las organizaciones gubernamentales. Esta falta se constata en las dificultades de acceso a las fuentes del Gobierno, sobre todo desde los medios privados. Los periodistas explican cómo el flujo informativo institucional no se adapta a las necesidades relacionadas con la inmediatez que caracteriza en la actualidad a la profesión periodística. Los entrevistados también acusan presiones desde los organismos públicos, focalizados fundamentalmente, en la Ley Orgánica de Comunicación. Dicha ley, obliga a los periodistas a amoldarse a una nueva forma de construir sus relatos informativos que aumenta la exigencia debido a las nuevas responsabilidades derivadas de posibles sanciones.

En México, la situación parece ser más extrema. Basalmente, es similar a lo expresado por otros países respecto a la dinámica político-empresarial y el clientelismo o amiguismo político. Sin embargo, la diferencia fundamental es la intensidad de la violencia y represión a la que podría estar sujeto un periodista, con el Gobierno como principal agente coactivo. Lo político como elemento condicionante en el periodista mexicano, comparativamente, es el caso más radical de presión política, entendida como represión de Estado. El reconocimiento de gran cantidad de sujetos de la situación de riesgo que se vive al investigar fuera de los márgenes establecidos define un escenario preocupante.

Y en el terreno de las audiencias, hay una dicotomía sobre la imagen de los periodistas, compuesta por el estereotipo de chismoso o vendido por una parte, pero al mismo tiempo valiente, por ejercer su labor en un contexto complejo. La conflictiva etapa que atraviesan los periodistas mexicanos afecta también la percepción que las audiencias tienen de ellos y, en consecuencia, del contenido que producen. Sin embargo, a pesar de ser un factor que incide en cómo se elabora la noticia, la presión social no es la condicionante principal de la rutina periodística en México.

Como puede observarse, los periodistas y los medios deben lidiar con una audiencia que exige un periodismo de calidad, aun cuando no exista una idea clara de lo que se entenderá por él. Surge allí una contradicción notoria: aparece una audiencia crítica y demandante de contingencia, pero al mismo tiempo un grupo que exige contenidos lúdicos y/o morbosos, lo que podemos llamar infoentretenimiento, que presentan altos niveles de consumo y sustentan publicitariamente los medios. Entonces, surge una peligrosa injerencia en la construcción de la agenda temática de los medios, no solo de la audiencia en términos globales a través de las redes sociales y otros soportes, sino también de grupos organizados, como agrupaciones sociales y ciudadanas, agencias de noticias, gabinetes de relaciones públicas y otros, que pugnan por influir, por poner sus temas en la contingencia y participar así en la delimitación del escenario informativo. Es justamente en esa presión donde existe un enorme riesgo.

En definitiva, las influencias que proceden del sistema (políticas y de presión social) muestran un tipo de relación similar, entre periodistas y políticos. Se produce una relación adversarial entre periodistas y políticos, cuando no se comparten los puntos de vista, o colateral, cuando el punto de vista es común entre ambos actores. Lo que diferencia a las diferentes zonas geográficas son las consecuencias de este tipo de relaciones. Así, en el cono sur, Argentina y Chile, la relación adversarial/colateral provoca una fuerte polarización de los medios de comunicación. En la zona andina, Ecuador y Colombia, la relación adversarial entre políticos y periodistas redunda en una 
falta de transparencia informativa por parte de las instituciones gubernamentales. Por último en México, las consecuencias del desarrollo de este modelo, van más allá, se traspasa la frontera de la transparencia, llegando a la agresión contra los periodistas. Puede observarse, también que a medida que se agravan las consecuencias de este tipo de relación, el peso otorgado por los periodistas a este condicionante aumenta. Así, la presencia de los condicionantes políticos, es menor en Argentina y Chile, que en México o Ecuador, donde el modelo adversarial conlleva sanciones a periodistas y medios de comunicación o agresiones físicas.

Una consecuencia de este modelo de relación, es que las audiencias de los diferentes países perciben a los periodistas en función de la adscripción política del medio, de tal forma, que esa relación periodistas/políticos condiciona la propia percepción de los periodistas por parte de las audiencias, perpetuando también en esta relación el modelo adversarial. Además, en muchas ocasiones, los periodistas idealizan los intereses de sus audiencias, adoleciendo de un contacto directo que evidencie dicha visión. La importancia de las audiencias en el trabajo periodístico, varía entre países de la misma zona geográfica, por lo que no podemos observar relación con cuestiones supranacionales, sino que responde a la sociología de los periodistas de cada país.

Esta radiografía nos lleva a plantear que las frecuentes transformaciones y vicisitudes que viven los periodistas, afectan directamente su labor y configuran un escenario que restringe el desempeño profesional y, por lo tanto, limita el rol social esperable de los informadores en todo sistema democrático. Ello permite plantear, para la discusión, las limitaciones actuales para el periodismo, o lo que hemos denominado "periodismo condicionado", como punto de inicio del más amplio debate a nivel profesional, académico y social. En este contexto, las propuestas reflexivas apuntan a otorgar un rol protagónico a las entidades formadoras de profesionales, por la relación directa que tienen con la situación actual del periodista. Sin embargo, este rol no apunta necesariamente a la limitación de la oferta informativa (pues el propio mercado debería regular los niveles de saturación dentro de la profesión), sino a que haya una intervención desde la perspectiva social; es decir, establecer las bases para una mayor coherencia entre la profesionalización de la actividad periodística y las verdaderas posibilidades de su ejercicio en la sociedad actual. Allí es donde está el desafío.

\section{Referencias}

1. Alonso, J. (2010). Cibermedios: conceptualización y tipologías. En M.A. Cabrera (ed.) Evolución tecnológica y cibermedios (pp. 21-38). Comunicación Social: Zamora.

2. Barbero, M. (2003). La globalización en clave cultural. Una mirada latinoamericana. Renglones, (53), pp.18-33. Recuperado de http://renglones.iteso.mx/upload/archivos/53_02_globalizacion.pdf

3. Casero, A. (2006). La crisis política como fenómeno comunicativo. La emergencia del caso crítico, Sphera Publica, (6), 179-192. Recuperado de http://sphera.ucam.edu/index.php/sphera-01/issue/view/7

4. Casero, A.. (2008). Modelos de relación entre periodistas y políticos: La perspectiva de la negociación constante. Estudios sobre el mensaje periodístico, (14), 111-128. Recuperado de https://revistas.ucm.es/ index.php/ESMP/issue/view/ESMP080811/showToc

5. Domingo, D. (2006). Inventing online journalism. Universitat Autónoma de Barcelona.

6. Eco, U. \& Fabbri, P. (1978): Progetto si ricerca sull'utilizzazione dell'informazione ambientale. Problemi dell' Informazione, 3 (4), 555-597. 
7. Gandy, O. (1982). Beyond the Agenda-Setting. Information Subsidies and Public Policy. Nueva Jersey: Ablex.

8. García-Canclini, N. (2001). Culturas híbridas. Estrategias para entrary salir de la modernidad. Buenos Aires: Paidós.

9. García de Torres, E., Yezers 'ka L., Rost A., Calderín M., Edo C., Rojano M. ... Corredoira L. (2011). Uso de Twitter y Facebook por los medios iberoamericanos. El profesional de la información, 20 (6), 611-620. Recuperado de http://www.elprofesionaldelainformacion.com/contenidos/2011/noviembre/02.pdf

10. Grossi, G. (1985). Rappresentanza e rappresentazione. Milano: Franco Angeli.

11. Guerrero, M. \& Márquez, M. (2014). El modelo "liberal capturado" de sistemas mediáticos, periodismo y comunicación en América Latina. Temas de Comunicación. (29), 135-170. Recuperado de http://revistasenlinea.saber.ucab.edu.ve/temas/index.php/temas/issue/view/N\%C2\%BA29\%20\%282014\%29

12. Hanitzsch, T., Anikina M., Berganza M., Cangoz I., Coman M., Hamada B. ... Wang Yuen K. (2010). Modeling perceived influences on journalism: evidence from a cross-national survey of journalists. Journalism \& Mass Communication Quarterly, 87(1), 5-22. Doi: http://dx.doi.org/10.1177/1077699 01008700101

13. Igartua, J.J., \& Humanes, M.L. (2004). Teoría e investigación en comunicación Social. Madrid: Síntesis.

14. Inglehart, R., \& Carballo, M. (1997). Does Latin America exist? (And is there a Confuncian culture?). A global analysis of cross-cultural differences. PS: Political Science \& Politics, (30), 34-47. Recuperado de http:// pol417.weebly.com/uploads/9/6/8/2/9682732/inglehart--confucian_culture.pdf

15. Kennamer, J. D. (Ed.). (1994). Public opinion, the press, and public policy. California: Greenwood.

16. Krippendorff, K. (1990) Metodología de análisis de contenido. Teoría y práctica. Barcelona: Paidós.

17. Lasswell, H. D. (1948). The structure and function of communication in society. The communication of ideas, (37), 215-228.

18. Manheim, J. (1994). Strategic Public Diplomacy and American Foreign Policy. The Evolution of Influence. Nueva York: Oxford University Press.

19. Martínez Albertos, J. (1999). El periodismo en el siglo xxl: más allá del rumor y por encima del caos. Estudios sobre el Mensaje Periodístico, 5, 15-35. Recuperado de http://revistas.ucm.es/index.php/ESMP/article/ view/ESMP9999110015A/12968

20. McChesney, R. (2004). The problem of the media: U.S. Communication Politics in the twenty-first century. New York: Monthly Review Press.

21. Mellado, C. (2009). Periodismo en Latinoamérica: Revisión histórica y propuesta de un modelo de análisis, Comunicar, (33), 193-201. Doi: http://dx.doi.org/10.3916/c33-2009-01-011

22. Messner, M., Linke, M., \& Eford, A. (2011). Shoveling tweets: an analysis of the microblogging engagement of traditional news organizations. In International Symposium on Online Journalism, Austin, Texas. Recuperado de https://online.journalism.utexas.edu/2011/papers/Messner2011.pdf

23. Oller, M., \& Chavero, P. (2015). La percepción de los factores de influencia de los periodistas dentro de la cultura periodística de Ecuador. Anuario Electrónico de Estudios en Comunicación Social "Disertaciones", 8(1), 81-104. Doi: dx.doi.org/10.12804/disertaciones.01.2015.04

24. Oller, M., \& Meier, K. (2012). La cultura periodística de España y Suiza. Madrid: Fragua.

25. Pérez, C. (2008). Comunicación política. Un reto para la especialización deperiodistas y fuentes. Ámbitos, 17, 251-269. Recuperado de http://issuu.com/ambitoscomunicacion/docs/revista-comunicacion-ambitos-17?e=0 
26. Ramonet, I. (2011). La explosión del periodismo. Buenos Aires: Capital intelectual.

27. Reese, S. (1999). Hacia una comprensión del periodista global. El modelo de investigación de jerarquías de influencia. Comunicación y Sociedad, 12 (2), 47-68. Recuperado de http://dadun.unav.edu/bitstream/10171/8793/1/20100226104850.pdf

28. Rodrigo-Alsina, M. (2005). La construcción de la noticia. Barcelona: Paidós.

29. Rost, A. (2014). Interatividade: Definições, estudos e tendencias. En J. Canavilhas (ed.). Webjornalismo: 7 caraterísticas que marcam a diferença (pp. 53-88). Covilha, Portugal: цAвсом. Recuperado de http://www. livroslabcom.ubi.pt/pdfs/20141204-201404_webjornalismo_jcanavilhas.pdf

30. Schramm, W. (1982). Investigación acerca de la comunicación en los Estados Unidos. En W. Schramm (ed.). La ciencia de la comunicación humana (pp. 3-20). Barcelona: Grijalbo.

31. Serrano, P. (2012). Periodismo canalla. Los medios contra la información. Barcelona: Icaria.

32. Serrano, P. (2014). La comunicación jibarizada. Barcelona: Península.

33. Shoemaker, P., \& Reese, S. (1996). Mediating the Message. Theories of Influences on Mass Media Content. New York: Longman.

34. Shoemaker, P., \& Vos. T. (2009). Gatekeeping Theory. New York: Taylor \& Francis.

35. Tuchman, G. (1983). La producción de la noticia: estudio sobre la construcción de la realidad. México: Gustavo Gili.

36. Vargas, M. (1998, 8 de diciembre). Sirenas en el Amazonas. El País. Recuperado de http://elpais.com/diario/1998/12/08/opinion/913071608_850215.html 